\title{
The Mathematical Model of Space - Time and Gravity
}

\author{
Syed Sohail Ahmed
}

\begin{abstract}
This model predicts the details of Origination (the Big Bang), Expansion, Contraction, Termination (the Big Crunch) and Repetition (the Big Loop) of Space - Time.

It represents time as internally related to the system determined by the gravity.

It gives the relationship between the speed of light and Gravitational constant.

Further, Schwarzschild radius can be represented independently either of speed of light or Gravitational constant.

The Gravitational Constant is directly proportional to Planck's Length and inversely proportional to Planck's Mass.

It predicts the existence of the Multiverse or regions within the Universe.
\end{abstract}

\section{Keywords}

Space, Time, Gravity, Gravitational Constant, Speed of light, Planck's units 
I. Sohail's Hexagon

II. The Origination, Expansion, Contraction, and Termination of Space - Time

III. The Time

IV. Relationship between Newton's Gravitational Constant and the Speed of Light

V. Schwarzschild radius independent of:

a) Speed of Light

b) Gravitational Constant

VI. Relationship between Planck's units and Gravitational Constant

VII. Universe vs Multiverse

\section{Sohail's Hexagon}

Consider the Line of Reference (LOR)

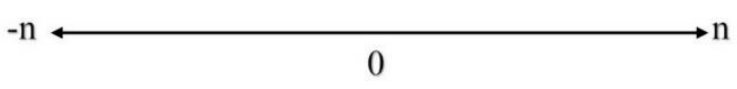

Figure 1

Positive $x$-axis and negative $x$-axis of length $n$

Extend a ray of length $\mathrm{n}$ from 0 and join both $\mathrm{x}$-axis and $\mathrm{y}$-axis.

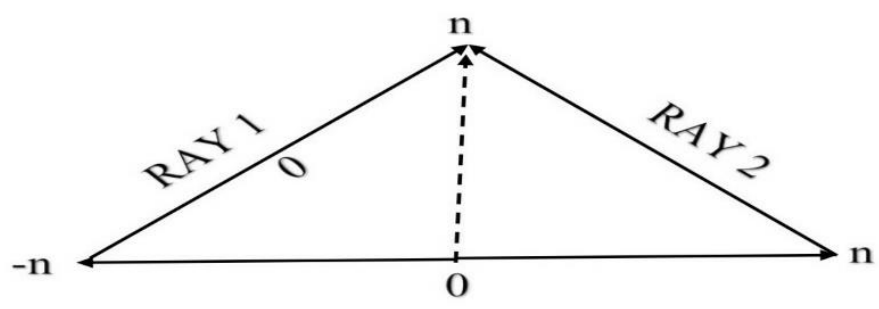

Figure 2

$y$-axis of length $n$ perpendicular to origin 0 is extended from origin 0 
Between negative $\mathrm{n}$ and positive $\mathrm{n}$, there is zero. Zero is absent between any two positive $\mathrm{n}$. If we see RAY 1, it is equal to Line of Reference (LOR). So, repeating the first step, we get back to same LOR.

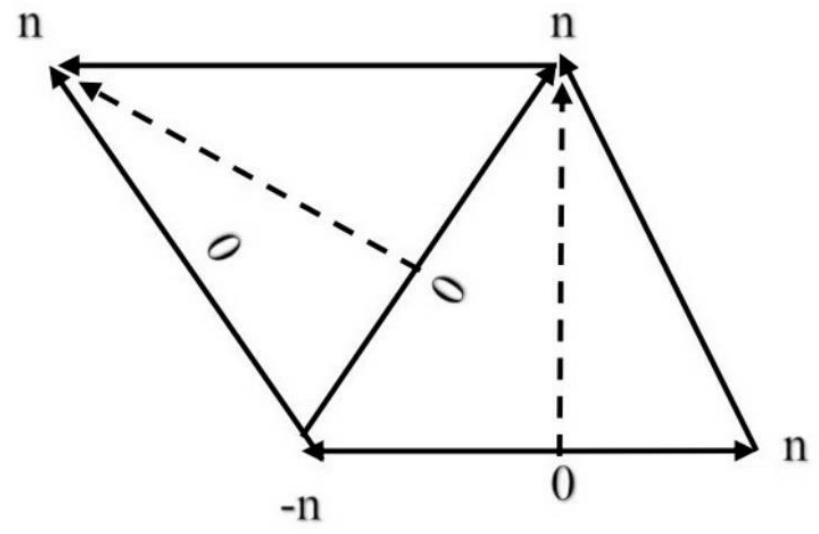

Figure 3

RAY 1 as new Line of Reference

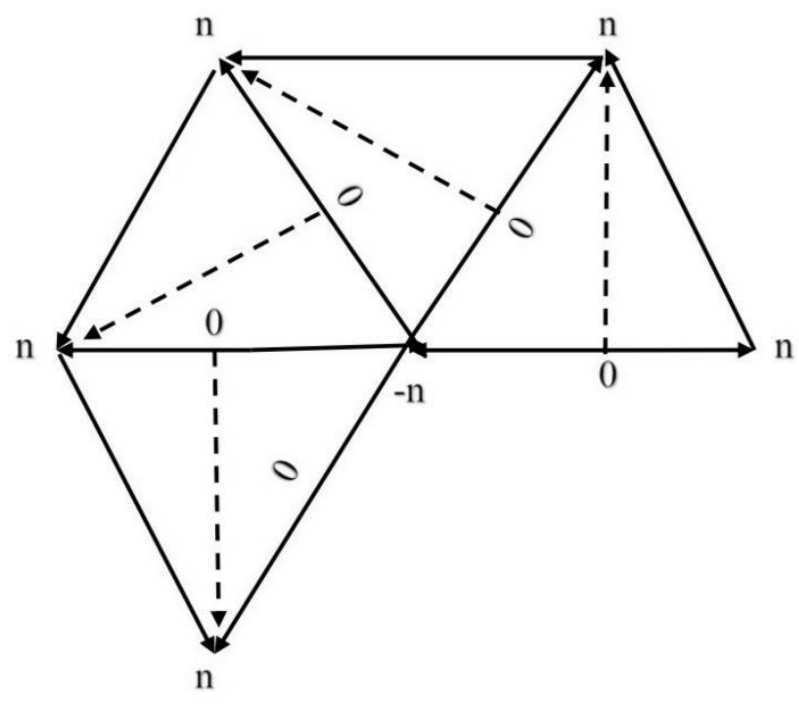

Figure 5

When extending a ray, always negative $n$ must be at left as it is defined at Line of Reference

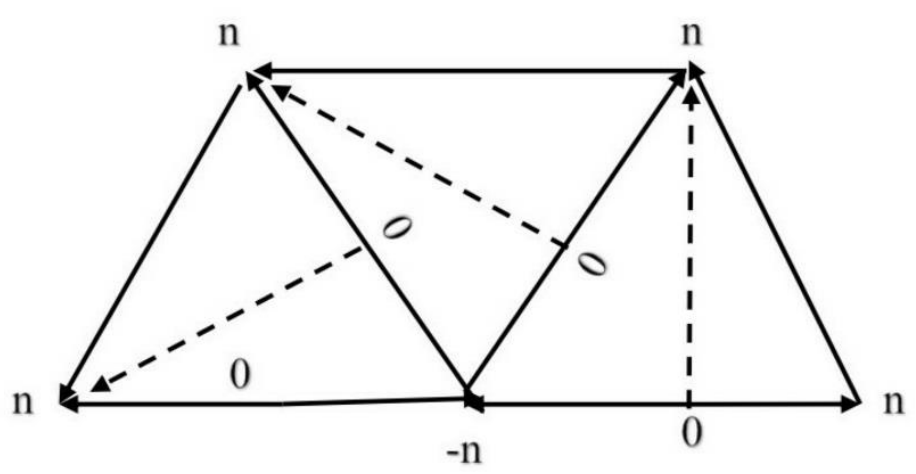

Figure 4

Half Phase Completion

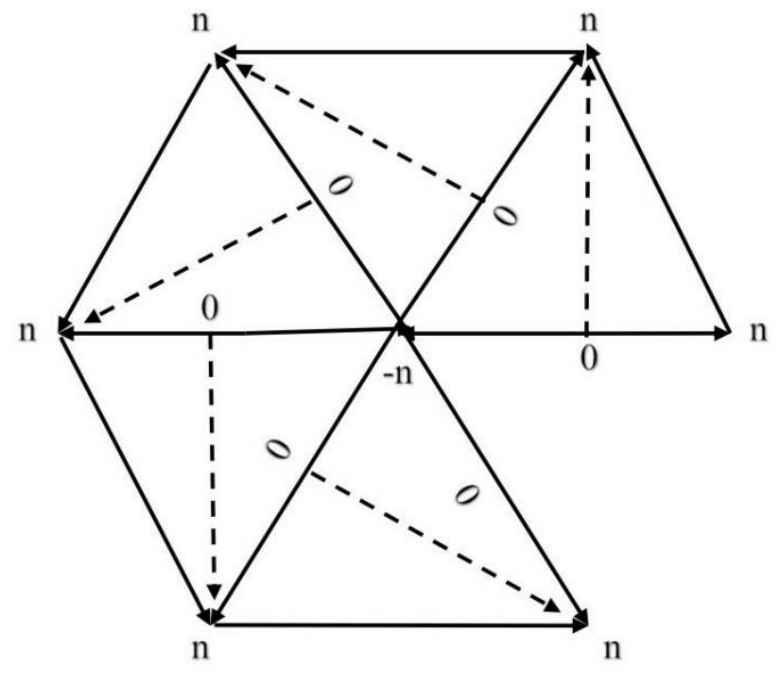

Figure 6

Negative $n$ is the point of rotation 


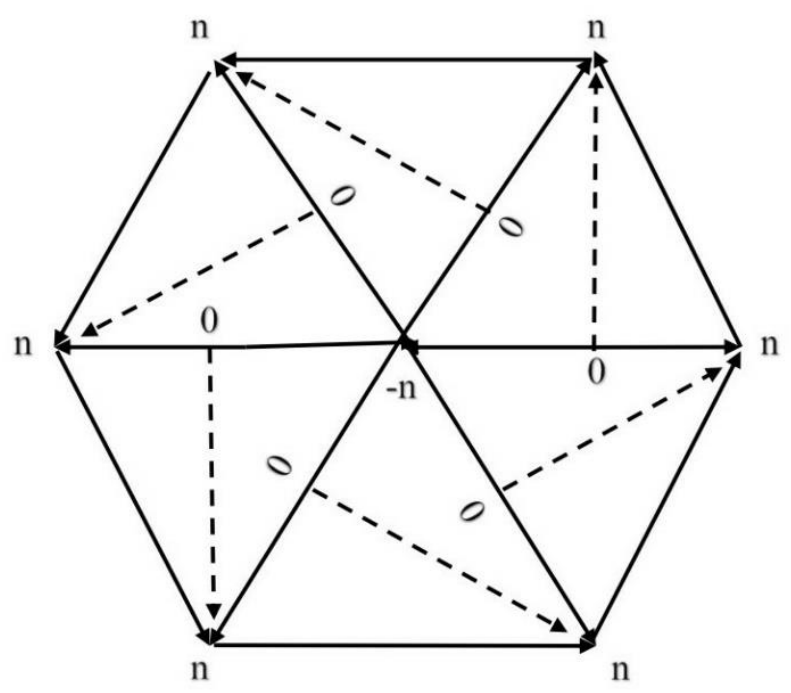

Figure 7

Sohail's Hexagon

At last, we get a regular hexagon of side length n. I refer it to as Sohail's Hexagon. Further, joining zeroes, we get:

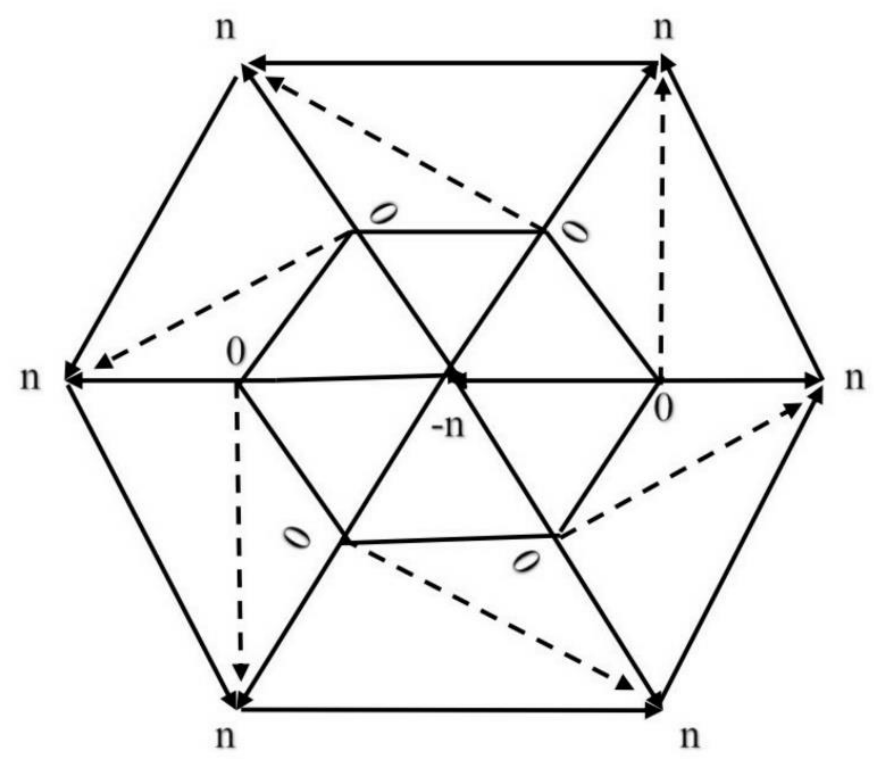

Figure 8

Sohail's Hexagon with connected zeroes.

It looks like a galaxy is enclosed within a hexagon.

It has 6 positive $n$ and only 1 negative $n$. 


\section{The Origination, Expansion, Contraction, Termination, and Repetition of Space - Time}

Consider a Sohail's hexagon of unit side length.

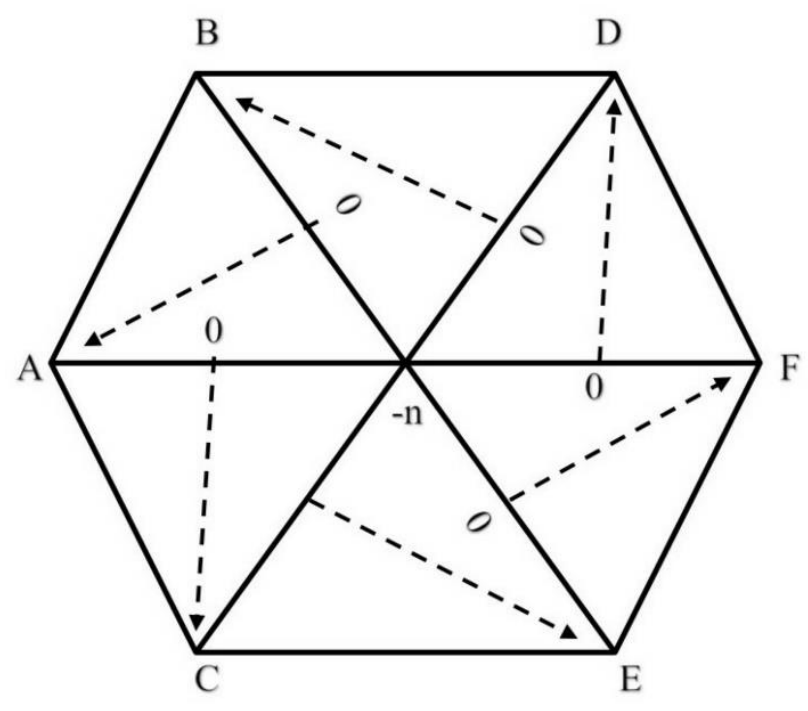

Figure 9

Sohail's Hexagon of unit side length

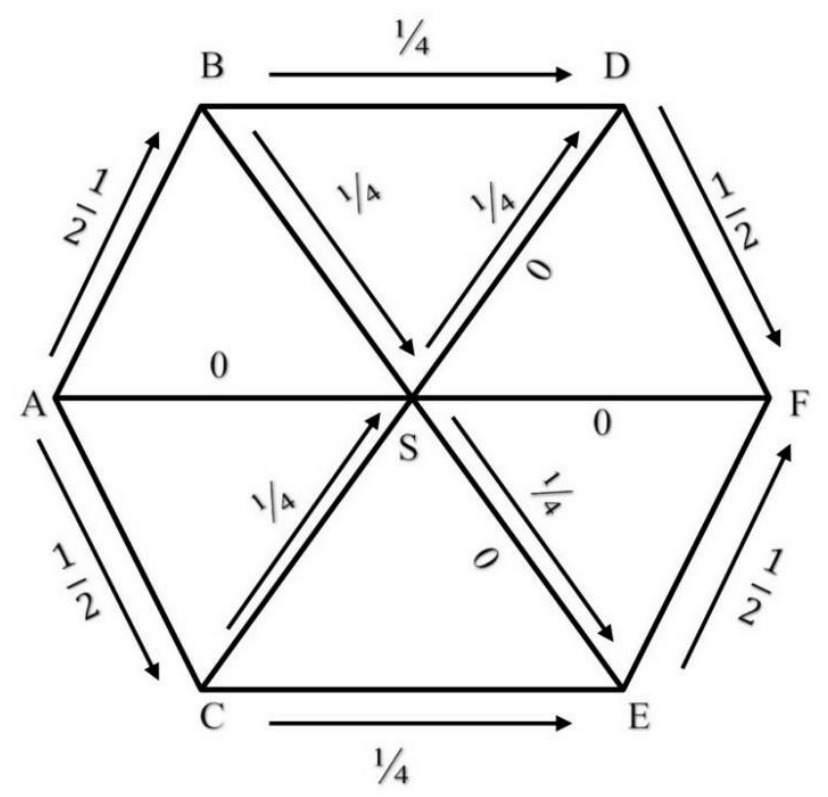

Figure 10

Split - Collision Model

$I^{\text {st }}$ Split (Origination, commonly referred to as The Big Bang):

Particle of mass 1 splits at A at an angle of $120^{\circ}$. At B and C, mass is $1 / 2$.

$2^{\text {nd }}$ and $3^{\text {rd }}$ Splits:

Further at B and C, particles split simultaneously at angles of $60^{\circ}$.

The Bounce:

At $S$, an ineffective collision occurs between half of B and half of $C$ (or two $1 / 4$ of initial mass) at an angle of $120^{\circ}$. So, they bounce off one another.

$1^{\text {st }}$ and $2^{\text {nd }}$ Collisions:

Half of 2nd Split collides with one of the bounced off particles at D. Half of 3rd Split collides with another bounced off particle at $\mathrm{E}$. 


\section{$3^{\text {rd }}$ Collision (Termination, commonly referred to as The Big Crunch):}

The final collision occurs at $\mathrm{F}$ at an angle of $120^{\circ}$. At $\mathrm{F}$, mass of a particle is 1 .

\section{The Big Loop:}

This model predicts the repetition of The Big Bang for at least once in the opposite arrow of time, so after the second Big Crunch, the particle is at the same place where it was at the first Big Bang.

\section{The Time}

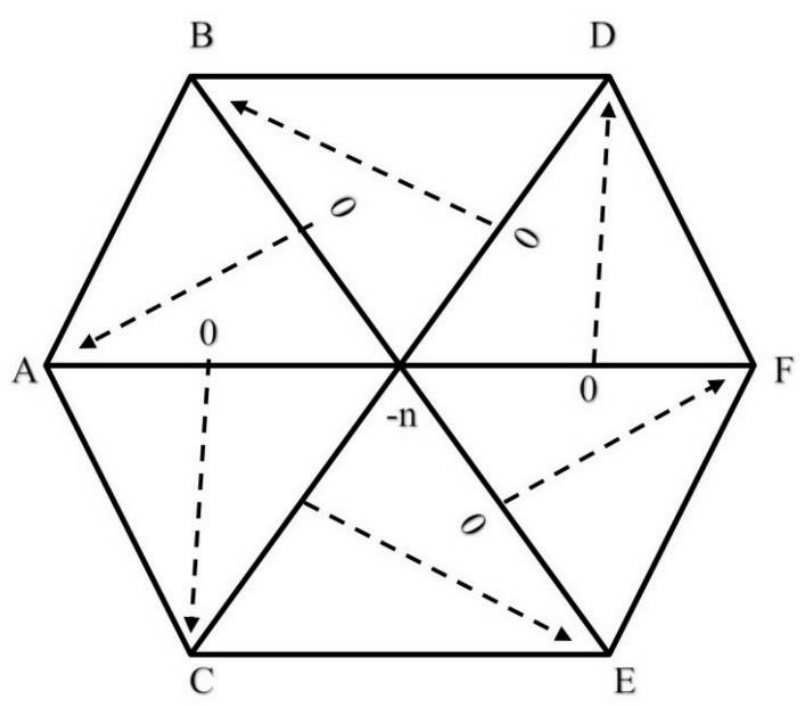

Figure 11
Consider Sohail's Hexagon as the system.

The diagonal AF represents the arrow of time.

Since, Space - Time originated simultaneously, the time is related to space internally of the system. To an internal observer, time is related.

And time is equal to the gravitational interaction of the particles.

Considering the Big Loop, after the second Big Crunch, to an external observer, it is as nothing has happened.

The Time is related internally of the system.

\section{Relationship between Newton's Gravitational Constant and the Speed of Light}

Consider Sohail's Hexagon of unit side length.

Length s from A (Origin) to $\mathrm{F}$ (End) is 2 units (length of diagonal of hexagon).

Rotation is anticlockwise. Angle between $\mathrm{A}$ and $\mathrm{F}$ is 0 . Let $\mathrm{m}$ be the mass of a particle at $\mathrm{A}$.

Work done is directly proportional to Energy. 
Work $\propto$ Energy

Energy $\propto$ Work

By mass - energy equivalence, Energy $E=m c^{2}$

Work done $W=F s \cos \theta$

$$
\begin{gathered}
\text { Force } F=m a \\
\text { Acceleration } a=\frac{s}{t^{2}}
\end{gathered}
$$

This Model defines time as defined by the Gravity.

$$
\text { Time } t=\text { Gravity } G
$$

$$
\begin{gathered}
E \propto W \\
m c^{2} \propto F s \cos \theta \\
m c^{2} \propto m a s(\cos 0) \\
m c^{2} \propto m a s(1) \\
m c^{2} \propto m\left(\frac{s}{t^{2}}\right) s(1)
\end{gathered}
$$

Mass $m$ on both sides gets cancelled

$$
\begin{gathered}
c^{2} \propto \frac{s^{2}}{t^{2}} \\
c \propto \frac{s}{t}
\end{gathered}
$$

Since $s=2, t=G$, equation becomes

$$
c \propto \frac{2}{G}
$$




$$
c=\frac{2}{G} K_{S}
$$

...Equation 1

Where:

- $\mathrm{c}=$ Speed of Light

$$
c=299792458 \frac{m}{s}
$$

- $\mathrm{G}=$ Gravitational Constant

$$
G=6.67430 \times 10^{-11} \frac{\mathrm{m}^{3}}{\mathrm{kgs}^{2}}
$$

- $\mathrm{K}_{\mathrm{S}}=$ Sohail's Constant

Sohail's Constant, $K_{S}=1.0004524012147 \times 10^{-2} S$

$$
S=\frac{\text { Jerk }}{\text { Density }}=\frac{j}{\rho}=\frac{\frac{m}{s^{3}}}{\frac{k g}{m^{3}}}=\frac{m^{4}}{k g s^{3}}
$$

Dimensionl Formula of S: $\left[L^{4} M^{-1} T^{-3}\right]$

Final equation:

$$
c=\frac{2}{G} \times\left(1.0004524012147 \times 10^{-2} S\right)
$$

Result:

- $\frac{c G}{K_{S}}=2 \rightarrow$ A dimensionless quantity 
V. Schwarzschild radius independent of:

a) Speed of Light

Schwarzschild radius, $r_{s}=\frac{2 G M}{c^{2}}$

$$
c^{2}=\frac{4}{G^{2}} \times K_{S}{ }^{2} \rightarrow \text { from equation } 1
$$

$$
\begin{gathered}
r_{s}=\frac{2 G M}{\frac{4}{G^{2}} \times K_{S}^{2}} \\
r_{s}=\frac{G^{3} M}{2 K_{S}^{2}}
\end{gathered}
$$

\section{b) Gravitational Constant}

$$
\begin{aligned}
c=\frac{2}{G} \times K_{S} \rightarrow \text { from equation } 1 & \\
G & =\frac{2}{c} \times K_{S} \\
r_{S} & =\frac{2 \frac{2}{c} K_{S} M}{c^{2}} \\
r_{S} & =\frac{4 K_{S} M}{C^{3}}
\end{aligned}
$$

$$
r_{s}=1.4852320538237331 \ldots \times 10^{-27} \times M
$$

Where:

- $\mathrm{M}$ is mass of object

- G is Newton's Gravitational Constant

- $\mathrm{C}$ is the Speed of Light

- $\mathrm{K}_{\mathrm{S}}$ is Sohail's Constant

$$
\begin{gathered}
K_{S}=1.0004524012147 \times 10^{-2} S \\
\left(1 S=1 \frac{\text { Jerk }}{\text { Density }}=1 \frac{\mathrm{m}^{4}}{\mathrm{kgs}^{3}}\right)
\end{gathered}
$$




\section{Relationship between Planck's units and Gravitational Constant}

Planck's length is given by

$$
l_{\mathrm{p}}=\sqrt{\frac{\hbar G}{c^{3}}}=1.616255 \times 10^{-35} \mathrm{~m}
$$

Planck's time is given by

$$
t_{\mathrm{P}}=\sqrt{\frac{\hbar G}{c^{5}}}=5.391247 \times 10^{-44} \mathrm{~s}
$$

The ratio of Planck's length square to Planck's time square is

$$
\begin{gathered}
\frac{l_{P}^{2}}{t_{P}^{2}}=\frac{\frac{\hbar G}{c^{3}}}{\frac{\hbar G}{c^{5}}}=c^{2} \\
c=\frac{l_{\mathrm{P}}}{t_{\mathrm{P}}} \\
c=\frac{2}{G} K_{S} \rightarrow \text { from equation } 1 \\
G=\frac{2}{c} K_{S} \\
G=\frac{2}{\frac{l_{\mathrm{P}}}{t_{\mathrm{P}}}} K_{S}
\end{gathered}
$$

Where:

$$
G=2 K_{S} \frac{t_{\mathrm{P}}}{l_{\mathrm{p}}}
$$

- G is Newton's Gravitational Constant

- $\mathrm{K}_{\mathrm{S}}$ is Sohail's Constant

$$
\begin{gathered}
K_{S}=1.0004524012147 \times 10^{-2} S \\
\left(1 S=1 \frac{\text { Jerk }}{\text { Density }}=1 \frac{\mathrm{m}^{4}}{\mathrm{kgs}^{3}}\right)
\end{gathered}
$$




\section{Universe vs Multiverse}

Consider Sohail's Hexagon with shaded regions

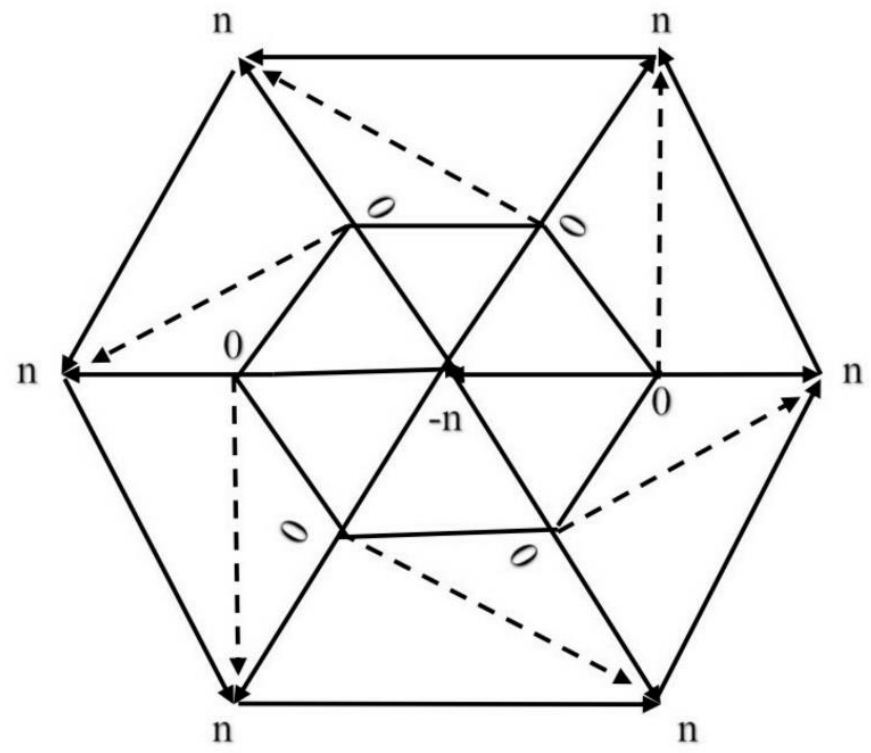

Figure 12 Sohail's Hexagon

There are 6 positive regions and 1 negative region.

Area of negative region is equal to all 6 positive regions.

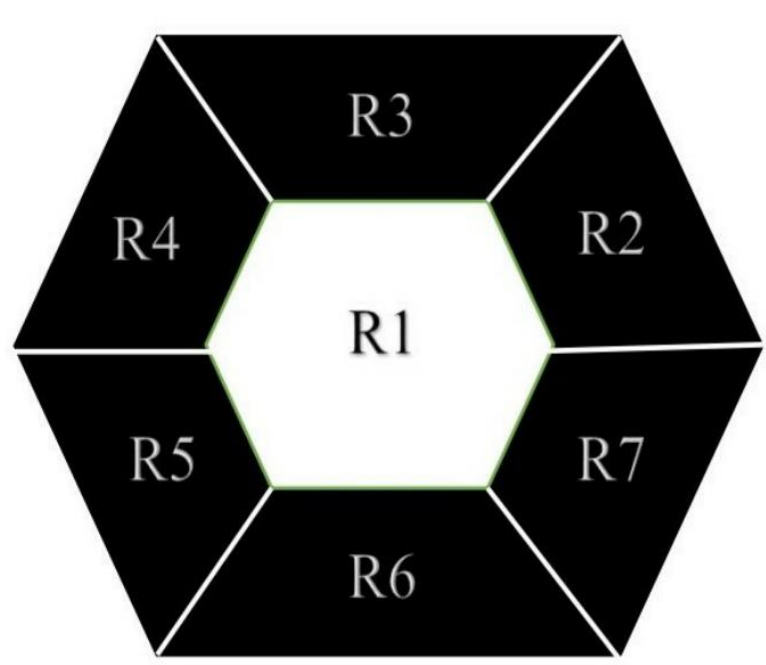

Figure 13

Black color represents positive regions from 0 to $n$. White color represents negative region from 0 to $-n$.

Predictions:

Either:

Our Universe has 7 regions with 6 positive regions revolving around 1 negative central region.

Considering position from centre, each region has variant physical laws with variant constants.

Or:

Our Universe is one of the seven universes in a multiverse.

Considering position from centre, each universe has variant physical laws with variant constants. 